\title{
Giant Lipoma of Forearm Causing Median Nerve Compression - A Case Report
}

\author{
Rachana DT, Hrishikesh PS (₫), Premashish JH \\ Department of Surgery, Jagjivanram Western Railway Hospital, Maratha Mandir Lane, Mumbai Central, \\ 400008 Mumbai, India.
}

\begin{abstract}
Giant lipomas in the proximal upper extremity are rare. Traditionally these are slow growing tumour and cause symptoms depending upon their size, position and compression of surrounding structures. Lipoma causing compression of median nerve in the forearm is rare. Surgical excision invariably leads to complete recovery. We present a case of a giant lipoma in forearm causing neuropathy of median nerve. It is difficult to clinically judge the extent of lipoma in atypical position and findings. MRI should be used in pre-operative evaluation of these cases. Surgical excision causes complete resolution of symptoms.
\end{abstract}

Keywords: $\quad$ Giant lipoma, liposarcoma, median nerve, nerve compression, neuropathy

\section{Correspondence:}

Hrishikesh P. Salgaonkar, Department of Surgery, Jagjivanram Western Railway Hospital, Maratha Mandir Lane, Mumbai Central, 400008 Mumbai, India. Tel: +65-90376065 Fax: +65-67746077 Email: hrishikesh.salgaonkar@gmail.com

\section{Introduction}

Lipomas are one of the commonest soft tissue tumors. They are slow growing, found in combination of varied tissue planes like subcutaneous, subfascial or intramuscular. Presentation may vary from asymptomatic to several pressure symptoms. Patients seek treatment due to compression symptoms or cosmetic deformities fearing social embarrassment. Lipomas commonly occur in extremities though ones causing symptomatic nerve compression in the forearm are rare.

Compressive neuropathy of median nerve is rare in proximal forearm or elbow joint and is mostly due to structural anomaly or anatomic variations e.g abnormalities of supracondylar process, insertion of bicipital aponeurosis, Ganzer's muscle or abnormal vasculature. Lipoma causing compressive neuropathy of median nerve is a rare finding. On literature review, we find few reports of lipomas affecting the median nerve, but the location in these reports is mainly in the hand and wrist $(1,2)$. We present a case of giant lipoma extending along the forearm with median nerve compression that was treated successfully.

\section{Case Report}

A 48-year-old male presented with a history of progressively increasing diffuse swelling over the volar aspect of the right forearm over a period of two years. The swelling was small, painless and not interfering with any activities. Patient denied any history of trauma or fever. Recent onset of parasthesia and numbness over right thumb and thenar eminence with weakness in movements of the right hand was the primary reason to seek medical opinion. He denied difficulty in doing fine movements like writing or key pinch, history of similar complaints in his left hand, associated neck pain or stiffness. No weakness found in movements at his elbow and shoulder joints.

On local examination, there was 20 x 6 x $5 \mathrm{~cm}$ diffuse swelling all along the flexor aspect of forearm. Overlying skin was normal, no dilated veins seen. Swelling was soft, non-tender and no rise in local 


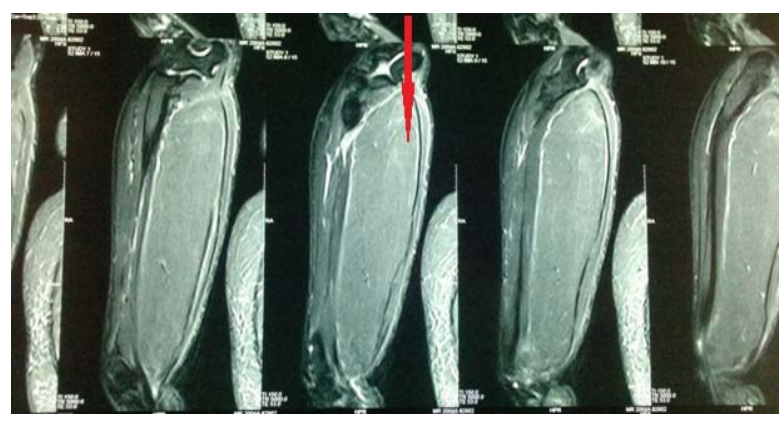

Figure 1: The tumour seen occupying the intermuscular plane of forearm on MRI.

temperature felt. No atrophy of thenar eminence was seen. On performing the static two point discrimination test, an area of hypoesthesia was found in the median nerve distribution in comparison to the contra lateral side. Phalen's test done was positive. Power was equal bilaterally on hand grip testing. No weakness in radial deviation of wrist and flexion of distal interphalangeal joint of index and ring finger. Peripheral pulsations and capillary refilling was normal.

A radiograph of the local part showed soft tissue swelling surrounding normal bony framework of right forearm. Electromyography testing along the median nerve distribution reported a prolonged sensory latency. On MR imaging, a large fat containing tumor $7.6 \times 7 \mathrm{~cm}$ (transverse) \& $25.7 \mathrm{~cm}$ in length, in volar compartment of the forearm, in the intermuscular planes between superficial \& deep flexor muscles was seen. No solid areas or focal area of enhancements noted. No obvious feeder was visualised but draining vessels were present. These findings were most likely to be a lipoma (Fig. 1).

Thereby, in view of nerve compression, excision of the mass was done under general anesthesia. A longitudinal incision was made along the entire volar aspect of forearm. After splitting of the superficial muscles of forearm a large encapsulated adipose containing mass was separated all over the wrist to the elbow joint (Fig. 2). The median nerve was seen enveloping the tumor all along its course and was meticulously dissected off. A drain was placed which was removed after 48 hours. On table the tumor weighed 765 grams and measured $24 \times 8 \times 5 \mathrm{~cm}$. Histopathological diagnosis confirmed presence of mature adipocytes suggestive of lipoma with no atypical cells. Post-operatively patient underwent active physiotherapy and recovered well with full motor movements.

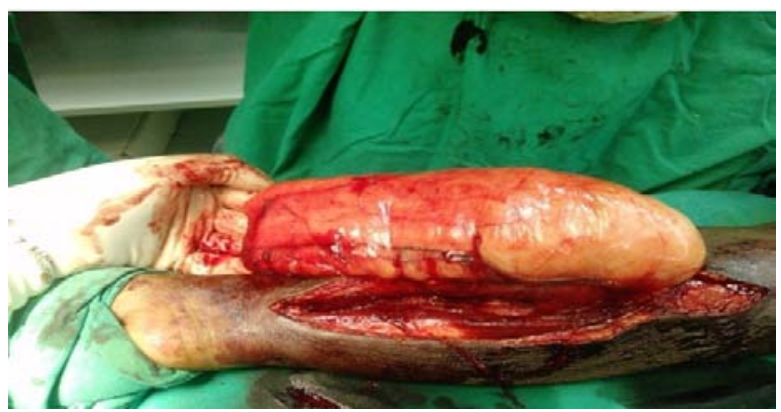

Figure 2: The tumour occupying the entire forearm after splitting the superficial muscles.

\section{Discussion}

Lipomas are adipose containing commonest mesenchymal tumours with an incidence of nearly $16 \%$ (3). They may occur commonly in subcutaneous tissues, intermuscular, intramuscular or paraosteal location. The clinical picture also varies according to the location. Large internal and deep seated lipomas e.g intra-abdominal or retro-peritoneal, intramuscular etc are known to cause complications. Intra-abdominal lipomas when large may produce abdominal pain, pressure effect leading to renal failure, intestinal obstruction. While intramuscular or lipomas near joint line can restrict the range of movements at the joint. Lipomas particularly in the retro-peritoneum, shoulder/scapular region and thigh region are known to undergo a sarcomatous change (4). In contrast, cutaneous lipomas are primarily a cosmetic problem, present as small subcutaneous swellings without any specific symptom. But occasionally these can cause functional limitation or lymph edema (4). Lipomas of more than $5 \mathrm{~cm}$ diameter aka Giant lipomas although rare can present in the thigh, shoulder or trunk. Giant upper limb lipomas are very rare (5). Clinical features of these giant lipomas are mainly due to their size and may include pain from stretching or compression of adjacent nerves, compartment syndrome, and restriction in movements of the joint involved. They may also cause social embarrassment or inability to wear clothing. Swellings in the upper limb are known to be symptomatic at small size itself. Lipomas in upper limb causing compressive neuropathy are uncommon (6). But subfascial and intramuscular lipoma are situated deep in close proximity to neurovascular bundles. They are known to cause compressive neuropathy (7).

On literature review we found case reports of lipoma causing compressive neuropathy of radial nerve in upper limb $(1,2,8)$ and also few cases involving the 
ulnar nerve compression in the forearm, wrist and palm (9). Median nerve compression has also been reported in the brachial plexus area's (10) and particularly in the wrist and the palmar region $(9,11,12)$. But only two cases of median neuropathy due to extrinsic compression by lipoma in forearm have been reported. In both cases the lipoma was located in proximal forearm with a clinical picture of anterior interosseous syndrome $(13,14)$. Cribb et al. (5) in there series reported 10 cases of giant lipomas causing neuropathy in upper limb. On histology 7 were lipomas, 2 cases were well differentiated liposarcoma and one neural fibrolipoma. Of these only two cases showed positive sign of median compression. While in one cases the lipoma was present in the hand, in the other case the location was not clarified. But this study suggested that it is important to evaluate large soft tissues tumours extensively. They also stress that it is necessary to perform a magnetic resonance imaging (MRI) in these cases routinely. It is important to make use of imaging methods particularly in large tumours and tumours located in inaccessible or atypical areas. An ultrasonography, computed tomography and MRI, and in rare cases when in doubt a technetium-99 diethylenetriaminepentaacetic acid scanning can be performed to confirm the diagnosis. In cases where these are inconclusive or in those patients who are non-compliant to these investigation (e.g. claustrophobia during MRI), a biopsy may be suggested.

Always keep a high index of suspicion for foci of sarcomatous changes in large lipomas and when in doubt, further evaluation is required. Lipomas located in atypical and inaccessible locations of the body (e.g. perineurallipoma, intramuscular lipoma, retroperitoneal lipomas) or those with atypical features (e.g. spindle cell, pleomorphic lipoma, chondroidlipoma, cellular angiolipoma, hibernoma) may be confused with liposarcomas adding to diagnostic uncertainty. Cytogenetic studies on the specimen can be helpful in distinguishing many of the lipoma variants. Solitary lipomas commonly have simple translocations or loss of chromosomal material involving chromosome 6,12 and 13. These changes are not seen in multiple lipomas or in spindle cell or pleomorphic lipomas. It is important that the pathologist examines multiple areas in the specimen as there may be a hidden foci of liposarcoma interspersed within well differentiated areas. Liposarcomas are one of the commonest adult soft tissue sarcoma. They have immature fat cells or lipoblasts on histology. The nucleus of these cells is hyperchromatic and eccentrically located. Presence of fat vacuoles on the nucleus can cause indentation or give it a scalloped appearance. A definitive diagnosis of giant lipoma can only be made after a detailed histopathological examination when a liposarcoma has been ruled out.

Complete surgical excision is the standard treatment for large lipoma. Recently, minimal invasive procedures such liposuction or suction assisted lipectomy are being popularized for management of large lipomas (15). However, haematoma formation and incomplete clearance leading to recurrence has been reported particularly in giant lipomas. Efficacy and safety of use of these minimal invasive techniques in lipomas in atypical position is not proven. In a lipoma close to a major blood vessel or nerve we may end up causing more harm.

\section{Conclusion}

Extrinsic compression of median nerve by a tumour is rare. And even rare is compression leading to neuropathy of median nerve. It is difficult to judge the extent of lipoma clinically particularly when the site is unusual with atypical findings. Lipoma is considered a common condition and surgery is routinely performed by trainees and juniors in any surgical unit. A casual approach while evaluating large lipoma may lead to incomplete removal or inadvertent complications. It is prudent to include a radiological imaging particularly a MRI during pre-operative evaluation of such cases. Biopsy may be suggested in selected cases. Surgical excision causes complete resolution of the symptoms.

\section{References}

1. Fitzgerald A, Anderson W, Hooper G. Posterior interosseous nerve palsy due to parosteal lipoma. J Hand Surg Br 2002; 27(6): 535-7.

2. Lidor C, Lotem M, Hallel T. Parosteal lipoma of the proximal radius: a report of 5 cases. J Hand Surg Am 1992; 17(6): 1095-7.

3. Abkari I, Abidi AE, Latifi. M. Giant Lipoma of the third finger: a case report. Chir Main 2011; 30(2): 152-4.

4. Enzinger FM, Weiss SW. Soft tissue tumors. St Louis: CV Mosby, 1988, pp. 301-45.

5. Cribb GL, Cool WP, Ford DJ, Mangham DC. Giant lipomatous tumors of the hand and forearm. J Hand Surg Br 2005; 30(5): 509-12.

6. Phalen GS, Kendrick JI, Rodriguez JM. Lipomas of the upper extremity. A series of fifteen tumours in the hand and six tumours causing nerve compression. Am J Surg 1971; 121(3): 298-306. 
7. Barber KW, Bianco AJ, Soule EH, MacCarty CS. Benign extraneural soft-tissue tumors of the extremities causing compression of nerves. $\mathrm{J}$ Bone Joint Surg Am 1962; 44A: 98-104.

8. Bieber EJ, Moore JR, Weiland AJ. Lipomas compressing the radial nerve at the elbow. J Hand Surg Am 1986; 11(4): 533-5.

9. Flores LP, Carneiro JZ. Peripheral nerve compression secondary to adjacent lipomas. Surg Neurol 2007; 67(3): 258-63.

10. Weinzweig N, Browne EZ. Infraclavicular median nerve compression caused by a lipoma. Orthopedics 1988; 11(7): 1077-8.

11. Oster LH, Blair WF, Steyers CM. Large lipomas in the deep palmar space. J Hand Surg Am 1989; 14(4): 700-4.
12. Sonoda H, Takasita M, Taira H, Higashi T, Tsumura $\mathrm{H}$. Carpal tunnel syndrome and trigger wrist caused by a lipoma arising from flexor tenosynovium: a case report. J Hand Surg Am 2002; 27(6): 1056-8.

13. Higgs PE, Young VL, Schuster R, Weeks PM. Giant lipomas of the hand and forearm. South Med J 1993; 86(8): 887-90.

14. Valbuena SE, O'Toole GA, Roulot E. Compression of the median nerve in the proximal forearm by a giant lipoma: A case report. J Brachial Plex Peripher Nerve Inj 2008; 3: 17.

15. Nichter LS, Gupta BR. Liposuction of giant lipomas. Ann Plast Surg 1990; 24(4): 362-5. 